\title{
VALIDACIÓN DE UN MÉTODO ANALÍTICO EMPLEANDO CROMATOGRAFÍA LÍQUIDA DE ALTA EFICIENCIA PARA LA DETERMINACIÓN DE IBUPROFENO EN MEDIOS BIORRELEVANTES
}

\author{
Sandra M. Gómez, Jorge A. Martínez y Fleming Martínez* \\ Departamento de Farmacia, Facultad de Ciencias, Universidad Nacional de Colombia, Sede Bogotá, A. A. 14490, Bogotá D. C., Colombia
}

Recebido em 10/12/09; aceito em 23/3/10; publicado na web em 29/6/10

\begin{abstract}
VALIDATION OF AN ANALYTICAL METHOD BY LIQUID CHROMATOGRAPHY FOR DETERMINATION OF IBUPROFEN IN BIORELEVANT MEDIA. An analytical method by liquid chromatography has been proposed and validated to study the apparent solubility of ibuprofen in biorelevant dissolution media. The main properties of the studied media were $\mathrm{pH}$ values of 5.0 and 6.5 and the presence or absence of some natural surfactant agents. The parameters evaluated were specificity, linearity, precision, accuracy, and detection and quantification limits, as well as the drug stability under the analysis conditions. The developed method was useful to determine the apparent solubility of this drug as a function of temperature and surfactants concentration to demonstrate the validity of the Biopharmaceutics Classification System.
\end{abstract}

Keywords: biorelevant media; HPLC; ibuprofen.

\section{INTRODUCCION}

La vía oral ha sido por excelencia la ruta más empleada para la administración de medicamentos, por ser la menos invasiva y más tolerada por el paciente. ${ }^{1}$ Sin embargo, el paso del fármaco hasta su sitio de absorción depende de factores fisiológicos propios del tracto gastrointestinal (como el peristaltismo, el volumen, el pH y la composición de los fluidos, el vaciado gástrico y, el estado patológico del paciente, entre otros) y de factores relacionados con las propiedades fisicoquímicas del fármaco (el pKa, el coeficiente de reparto, la solubilidad, y la lipofilicidad, entre otros), sin olvidar los aspectos relacionados con la forma de dosificación. Todos estos factores afectan la velocidad y la cantidad total de fármaco absorbido. ${ }^{2}$

Los parámetros que determinan la absorción de un fármaco después de su administración oral son la solubilidad y la permeabilidad. La solubilidad es uno de los factores más importantes que afecta la absorción intestinal de fármacos, puesto que solamente los compuestos que están en solución en el sitio de absorción pueden atravesar la membrana intestinal y alcanzar la circulación general. ${ }^{3}$ La solubilidad adquiere importancia particular en el caso de fármacos poco solubles, que por sus características estructurales suelen presentar valores de solubilidad en el agua inferiores a los que se pueden presentar bajo las condiciones fisiológicas. Por esta razón, ha sido necesario el estudio de la solubilidad de estos fármacos en medios comúnmente denominados biorrelevantes, que presentan valores de pH similares a los encontrados en el estómago y el intestino y que cuentan con otros compuestos que actúan como surfactantes biológicos, como las sales biliares y los fosfolípidos. ${ }^{4}$

Los medios biorrelevantes, fueron desarrollados inicialmente para mejorar la evaluación de las características de disolución in vitro que reflejan el desempeño in vivo de las formas farmacéuticas orales cuando estas son utilizadas. ${ }^{5,6}$ Dado que estos medios contienen características que los hacen más similares a los fluidos fisiológicos gastrointestinales, han sido útiles para establecer correlaciones entre la disolución del fármaco en una forma farmacéutica y los niveles plasmáticos que se aprecian luego de su absorción. Dos de estos medios denominados FaSSIF (Fasted State Simulated Intestinal Fluid) y FeSSIF (Fed State Simulated Intestinal Fluid), se caracterizan

\footnotetext{
*e-mail: fmartinezr@unal.edu.co
}

porque contienen sales biliares, fosfolípidos, el $\mathrm{pH}$, la osmolalidad y la tensión superficial en valores similares a los que se presentan en el intestino delgado antes y después de la ingesta de alimentos. ${ }^{5,6}$

Otra razón para estudiar la solubilidad de fármacos en el tracto gastrointestinal se debe a la importancia que este parámetro junto con la permeabilidad, presentan en la clasificación de fármacos de acuerdo al Sistema de Clasificación Biofarmacéutico (SCB). Esta clasificación proporciona una oportunidad para la exención de los estudios de bioequivalencia in vivo de productos de liberación inmediata que deseen la intercambiabilidad terapéutica con el producto innovador. La clasificación en el grupo de fármacos de alta solubilidad y alta permeabilidad (Clase I), de algunos ácidos débiles como los antiinflamatorios no esteroidales (AINEs), se restringe cuando al hacerse los estudios de solubilidad respectivos estos compuestos son insolubles en medios acuosos a $\mathrm{pH}$ 1,2 y 4,5. Estos resultados han planteado sugerencias respecto a las exigencias de las entidades regulatorias de algunos países como Estados Unidos, entre las que se encuentran la realización de estudios de solubilidad y de disolución empleando fluidos gástricos e intestinales reales o medios de disolución biorrelevantes ${ }^{7}$ y la determinación de la solubilidad únicamente en medios acuosos que presenten $\mathrm{pH}$ superiores a 5, para este tipo de compuestos. ${ }^{8}$ Algunas de las sugerencias han sido tenidas en cuenta, y se han desarrollado nuevos lineamientos que presentan menores restricciones para algunos compuestos con estas características. ${ }^{9}$

El Ibuprofeno (IBP, Figura 1) ${ }^{10}$ es un antiinflamatorio no esteroidal (AINE), y reconocido inhibidor no selectivo de la ciclooxigenasa (COX) ${ }^{11}$ Es principalmente utilizado como analgésico y antiinflamatorio en el tratamiento de la osteoartritis y la artritis reumatoidea, entre otras enfermedades. También presenta propiedades antipiréticas. El IBP es considerado como uno de los compuestos más seguros dentro de los de su clase.<smiles>CC(C)Cc1ccc(C(C)C(=O)O)cc1</smiles>

Figura 1. Estructura molecular del ibuprofeno

De otro lado, se ha establecido la monografía que incluye información científica que soporta la bioexención de estudios de 
bioequivalencia de los productos de liberación inmediata que contengan IBP, dada la amplia ventana terapéutica, las indicaciones farmacológicas, y su absorción completa que hacen de este fármaco un prototipo ideal para este tipo de excepción a pesar de no ser de Clase I de acuerdo al SCB y presentar escasa solubilidad a valores bajos de $\mathrm{pH} .{ }^{11}$ Dado que el IBP presenta amplio uso y su solubilidad depende del $\mathrm{pH}$, es importante estudiar el efecto que tienen los componentes presentes en los medios biorrelevantes sobre su solubilidad, con el fin de predecir esta propiedad fisicoquímica bajo condiciones fisiológicas. La ampliación de la información acerca de la determinación de la solubilidad de compuestos de esta clase generará mayor soporte científico que justifique el uso de estos medios biorrelevantes en la determinación de la solubilidad, para la clasificación de este tipo de compuestos y de otros compuestos lipófilos que pertenecen a la clase II según el SCB.

Si bien, en la literatura se han presentado diferentes métodos analíticos para la cuantificación de IBP en productos farmacéuticos y en muestras biológicas, ${ }^{12}$ es de resaltar que hasta el momento presente, en la literatura no se han reportado métodos validados para la determinación de IBP en los medios biorrelevantes FaSSIF y FeSSIF. Por lo tanto, en el presente trabajo se validó un método por Cromatografía líquida de alta eficiencia (CLAE) para la cuantificación de este fármaco en estos medios biorrelevantes y en soluciones amortiguadoras de $\mathrm{pH}$ 5,0 y 6,5, con el fin de determinar la solubilidad aparente en condiciones similares a las encontradas fisiológicamente y así, poder compararla con la solubilidad obtenida en ausencia de surfactantes de origen natural como los fosfolípidos y las sales biliares. Los parámetros de especificidad, linealidad, precisión, exactitud, y estabilidad fueron establecidos para la validación. Adicionalmente se utilizó el método para determinar la solubilidad de IBP en medios FeSSIF y FaSSIF a $37,0^{\circ} \mathrm{C}$ y establecer la conveniencia del uso de estos medios en la clasificación de fármacos basado en el SCB.

\section{MATERIALES Y METODOS}

\section{Reactivos}

Ibuprofeno BASF Corporation (Texas, E.U.) y Ketoprofeno (KTP) (Ningbo Bulkchem Chemical Co., Ltd., Zhejiang, China) fueron verificados contra el estándar primario USP (United States Pharmacopeia) respectivo. ${ }^{13}$ Los dos fármacos presentaron una pureza superior al 0,995 en fracción másica. El Taurocolato de sodio (Figura $2)^{10}$ se obtuvo en Sigma Aldrich (St. Louis, MO, E.U.) y la fosfatidilcolina de soya (Figura 3) ${ }^{10}$ fue comprada en Spectrum Chemical Corp. (New Brunswick, NJ, E.U.). El Metanol y el Acetonitrilo fueron grado CLAE y obtenidos en Merck (Darmstadt, Alemania). El agua usada fue destilada y filtrada por membrana acetato de celulosa de 0,45 $\mu \mathrm{m}$ para cumplir con los requerimientos de CLAE. Los demás reactivos empleados en la preparación de las soluciones amortiguadoras fueron de grado analítico.

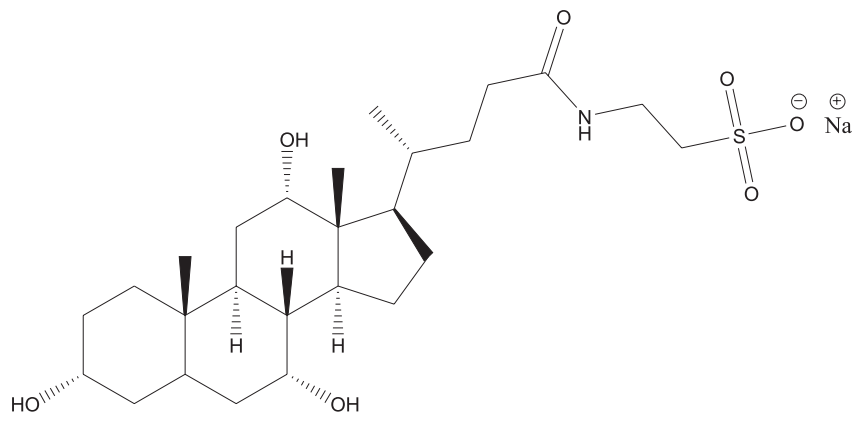

Figura 2. Estructura molecular del taurocolato de sodio

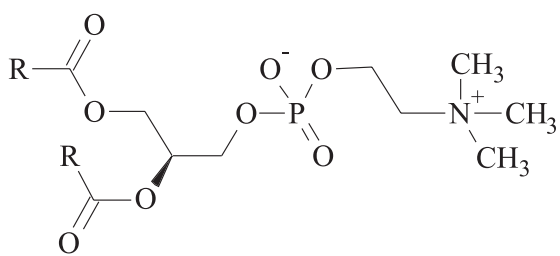

Figura 3. Estructura molecular de la lecitina. R corresponde a ácidos grasos, los cuales, en la lecitina de soya se distribuyen principalmente así: palmítico $11,7 \%$, esteárico $4,0 \%$, palmitoleico $8,6 \%$, oleico $9,8 \%$, linoleico $55,0 \%$, linolénico 4,0\%, otros ácidos $C_{20}$ a $C_{22}$ (incluyendo araquidónico) 5,5\%

\section{Equipos y condiciones analíticas}

Se empleó un cromatógrafo modular Agilent 1200 con inyector manual con un loop de $20 \mu \mathrm{L}$, bomba cuaternaria, detector de arreglo de diodos (DAD) e integrador Agilent Chem Station. Se utilizó una columna LiChroCART ${ }^{\circledR}$ Purospher ${ }^{\circledR}$ RP-18 encapada con tamaño de partícula de $5 \mu \mathrm{m}, 125 \mathrm{~mm}$ de longitud y $3 \mathrm{~mm}$ de diámetro interno y, un guardacolumna Lichrospher ${ }^{\circledR} 100$ RP-18e. Las muestras se eluyeron con una mezcla de $50 \%$ de acetonitrilo y $50 \%$ de agua de calidad CLAE ajustada a pH 3,0 con $\mathrm{H}_{3} \mathrm{PO}_{4}$ al $85 \%$ a un flujo de $1 \mathrm{~mL} \mathrm{~min}^{-1}$ y una temperatura de $35,0{ }^{\circ} \mathrm{C} .{ }^{14} \mathrm{La}$ cuantificación se estableció a una longitud de onda de $264 \mathrm{~nm}$. Para la determinación de la solubilidad de IBP en FeSSIF y FaSSIF a 37,0 ${ }^{\circ} \mathrm{C}$ se empleó un agitador mecánico Wrist Action Burrel modelo 75, un baño de calentamiento Julabo SW 23 con agitación y un potenciómetro Orion 2-Star Thermo Scientific. Las muestras fueron filtradas antes de inyectarse en el cromatógrafo a través de filtros HV Millex (Millipore Corporation, E.U.) de 0,45 $\mu \mathrm{m}$.

\section{Preparación de los medios}

La preparación de los medios FaSSIF y FeSSIF que simulan los fluidos intestinales antes y después de la ingesta de los alimentos se hizo acorde con las sugerencias de Dressman y colaboradores. ${ }^{3}$ Las soluciones amortiguadoras de $\mathrm{pH} 5,0$ y $\mathrm{pH}$ 6,5 se prepararon sin la adición de taurocolato y fosfatidilcolina. Se aseguró al final de la preparación el ajuste de $\mathrm{pH}$. En la Tabla 1 se indica la composición final de estos medios. ${ }^{2,6,15}$

Tabla 1. Composición de los medios biorrelevantes intestinales y de las soluciones amortiguadoras utilizados

\begin{tabular}{lcccc}
\hline Sustancia & $\begin{array}{c}\text { Solución } \\
\text { amortiguadora } \\
\text { pH 5,0 }\end{array}$ & $\begin{array}{c}\text { Solución } \\
\text { amortiguadora } \\
\text { pH 6,5 }\end{array}$ & FeSSIF & FaSSIF \\
\hline $\mathrm{NaCl}$ & $173 \mathrm{mM}$ & $106 \mathrm{mM}$ & $173 \mathrm{mM}$ & $106 \mathrm{mM}$ \\
$\mathrm{NaH}_{2} \mathrm{PO}_{4}$ & & $28,66 \mathrm{mM}$ & \multicolumn{2}{c}{$28,66 \mathrm{mM}$} \\
Acido acético & $144 \mathrm{mM}$ & & $144 \mathrm{mM}$ & \\
Taurocolato de sodio & & & $15 \mathrm{mM}$ & $3 \mathrm{mM}$ \\
Fosfatidilcolina & & & $3,75 \mathrm{mM}$ & $0,75 \mathrm{mM}$ \\
$\mathrm{NaOH}$ & $101 \mathrm{mM}$ & $13,8 \mathrm{mM}$ & $101 \mathrm{mM}$ & $13,8 \mathrm{mM}$ \\
\hline
\end{tabular}

\section{Validación del método analítico}

La validación del método se hizo teniendo en cuenta los lineamientos establecidos en la Conferencia internacional de armonización (ICH) y la farmacopea USP, analizando los siguientes parámetros: ${ }^{13,16}$

\section{Especificidad}

Para demostrar que la señal obtenida correspondía únicamente al analito IBP y no a impurezas o productos de degradación o componentes de los medios empleados, se prepararon soluciones madre del 
estándar de IBP con una concentración equivalente a $2000 \mu \mathrm{g} \mathrm{mL} \mathrm{m}^{-1}$ en el diluente, en las soluciones amortiguadoras de $\mathrm{pH} 5,0$ y 6,5 y en los medios FeSSIF y FaSSIF, de donde se tomó un volumen al que se adicionó estándar interno de KTP para tener una concentración final de 8 y de $300 \mu \mathrm{g} \mathrm{mL}^{-1}$ de IBP, llevando a volumen con diluente. Cada una de las muestras fue inyectada, así como los medios, el diluente y la fase móvil. Se determinó la pureza de pico del estándar de IBP y del estándar interno de KTP en cada una de las muestras.

Adicionalmente, para observar si los componentes del medio, o los productos de degradación que pudieran originarse bajo condiciones de estrés presentaban interferencias en el tiempo de retención del IBP, se prepararon soluciones madre de IBP en los medios FaSSIF y FeSSIF de los cuales se tomó una alícuota a la que se adicionó en cada caso: 1,0 mL de $\mathrm{HCl} 1,0 \mathrm{M}, 1,0 \mathrm{~mL}$ de $\mathrm{NaOH} \mathrm{1,0} \mathrm{M,} \mathrm{1,0}$ $\mathrm{mL}$ de $\mathrm{H}_{2} \mathrm{O}_{2}$ al $30 \%$ y $1,0 \mathrm{~mL}$ de $\mathrm{H}_{2} \mathrm{O}$ y se sometieron durante 1 h en un baño termostatizado a $60,0{ }^{\circ} \mathrm{C}$. A su vez, alícuotas de las soluciones madres preparadas, se expusieron durante $3 \mathrm{~h}$ a la luz UV a una longitud de onda de $254 \mathrm{~nm}$. Al final del ensayo todas las muestras se completaron a volumen con diluente para alcanzar una concentración correspondiente a $300 \mu \mathrm{g} \mathrm{mL}^{-1}$ de IBP. Se obtuvieron los cromatogramas de estas muestras, de un estándar sin degradar y de los respectivos blancos.

\section{Linealidad}

La linealidad fue evaluada preparando como mínimo tres soluciones madre del estándar de IBP con una concentración equivalente a $2000 \mu \mathrm{g} \mathrm{mL}^{-1}$ en el diluente, en las soluciones amortiguadoras de $\mathrm{pH}$ 5,0 y 6,5 y en los medios FeSSIF y FaSSIF. A partir de estas soluciones se prepararon 5 soluciones de trabajo con concentraciones entre 70 y $600 \mu \mathrm{g} \mathrm{mL}^{-1}$, a las que se adicionó $300 \mu \mathrm{L}$ de $\mathrm{HCl} 1,0 \mathrm{M}$ y un volumen de solución estándar de KTP para obtener en las muestras una concentración final de $8 \mu \mathrm{g} \mathrm{mL} \mathrm{m}^{-1}$. La evaluación de la linealidad se hizo a través del análisis de varianza (ANOVA) para la regresión, y el estadístico $t$ de Student se usó para evaluar la pendiente y el intercepto, asumiendo una confianza del $95 \%$.

\section{Precisión}

La precisión fue determinada a través de la evaluación de la repetibilidad (intradías) y de la precisión intermedia (interdías). En el caso de la repetibilidad se prepararon 3 soluciones madre con IBP en diluente, las soluciones amortiguadoras y los medios biorrelevantes y de cada una de ellas se hicieron tres diluciones a 70, 300 y 600 $\mu \mathrm{g} \mathrm{mL}^{-1}$ de IBP. La precisión se evaluó a través del coeficiente de variación, llamada también la desviación estándar relativa (\% RSD). La precisión intermedia fue determinada a través de los resultados obtenidos por 3 réplicas de concentración intermedia $\left(300 \mu \mathrm{g} \mathrm{mL} \mathrm{L}^{-1}\right)$ en cada uno de los medios, preparadas por analistas diferentes en 2 días diferentes. La precisión intermedia se evaluó mediante un ANOVA empleando una confianza del $95 \%$ para cada uno de los medios empleados.

\section{Exactitud}

La exactitud fue establecida mediante la relación de áreas de IBP y estándar interno obtenidas para 3 concentraciones $(70,300$ y $600 \mu \mathrm{g}$ $\mathrm{mL}^{-1}$ ) de IBP en las soluciones amortiguadoras y los medios biorrelevantes y su comparación contra un estándar de IBP de concentración conocida que fue inyectado 6 veces. Los valores obtenidos fueron luego expresados como porcentaje de recuperación. Se emplearon 3 réplicas en cada caso.

\section{Límite de cuantificación $(L C)$ y límite de detección $(L D)$}

Los límites de cuantificación y detección se determinaron a partir de la pendiente y la desviación estándar del intercepto de las curvas de calibración obtenidas para el sistema y el método es decir, para el diluente y las soluciones amortiguadoras, así como también los medios biorrelevantes.

\section{Estabilidad}

Con el fin de determinar la estabilidad del IBP en aquellas condiciones a las que estaría expuesto durante el ensayo de solubilidad, se prepararon 3 réplicas con una concentración de $300 \mu \mathrm{g} \mathrm{mL}^{-1}$ de IBP en cada uno de los medios y se almacenaron las muestras a temperatura ambiente durante $96 \mathrm{~h}$, a $4,0{ }^{\circ} \mathrm{C}$ durante $96 \mathrm{~h}$ y a 40,0 ${ }^{\circ} \mathrm{C}$ durante $48 \mathrm{~h}$. Las áreas de IBP determinadas al comienzo de cada ensayo se compararon con las obtenidas al final del ensayo con el fin de identificar su posible degradación.

\section{Determinación de la solubilidad de IBP en medios biorrelevantes}

Se adicionó IBP a viales que contenían $5 \mathrm{~mL}$ del medio, las muestras fueron sometidas a agitación mecánica durante $1 \mathrm{~h}$ y posteriormente ubicadas en el baño termostatizado a $37,0^{\circ} \mathrm{C}$ durante 48 h, manteniendo una agitación de $100 \mathrm{rpm}$. Cada muestra se filtró y recogió en un vial ámbar de $5 \mathrm{~mL}$, de donde se tomó una alícuota a la que le fue adicionado $300 \mu \mathrm{L}$ de $\mathrm{HCl} 1,0 \mathrm{M}$ y un volumen de solución de estándar interno de KTP para llevar a una concentración final de 8 $\mu \mathrm{g} \mathrm{mL}{ }^{-1}$, completando a volumen con diluente. Las jeringas, los filtros y los viales en donde se recolectaron las muestras fueron calentados previamente a la temperatura de estudio, con el fin de evitar la precipitación del fármaco. Posteriormente las muestras fueron inyectadas en el cromatógrafo líquido. Se hicieron como mínimo 3 réplicas.

\section{RESULTADOS Y DISCUSION}

El IBP presentó un tiempo de retención aproximado de 3,70 min y el KTP de 1,70 min, de modo que el análisis cromatográfico fue de $5 \mathrm{~min}$, haciendo de este un método rápido para la cuantificación de IBP. La idoneidad del sistema se evaluó mediante la simetría, el factor de coleo, el número de platos teóricos y la resolución obtenidos a partir de 10 inyecciones del estándar. Los resultados que se encuentran resumidos en la Tabla 2 indican desviaciones relativas menores o iguales a 2, mostrando la precisión en los valores de las áreas del estándar de IBP y el estándar interno. La resolución es mayor a 2,5 y el factor de coleo es menor a 2,5 para ambos estándares. Estos valores son reportados en la farmacopea norteamericana para establecer el sistema cromatográfico antes de proceder con la valoración de IBP. ${ }^{13}$

Tabla 2. Resultados obtenidos para de la idoneidad del sistema

\begin{tabular}{llllll}
\hline Fármaco & $\%$ RSD Áreas & $S$ & $T$ & $N$ & $r$ \\
\hline IBP & 2,0 & 0,9 & 1,1 & 3999,3 & 10,1 \\
KTP & 1,8 & 0,9 & 1,1 & 1699,4 &
\end{tabular}

RSD = desviación estándar relativa, $S=$ simetría, $T=$ factor de coleo ${ }^{11}, N=$ platos teóricos, $r=$ resolución

La Figura 4 presenta los cromatogramas obtenidos luego del análisis de las soluciones amortiguadoras, los medios biorrelevantes, el diluente y la fase móvil sin IBP o KTP. No se determinaron interferencias en los picos de los estándares cuando estos fueron adicionados a los medios o al diluente. El análisis de la pureza de pico determinó que los espectros tomados en la porción ascendente, máxima y descendente del pico correspondiente al IBP y al KTP son iguales, demostrando la especificidad del método en los diferentes medios utilizados.

En el caso de las muestras que fueron expuestas a condiciones de degradación no se apreciaron interferencias en el tiempo en que 


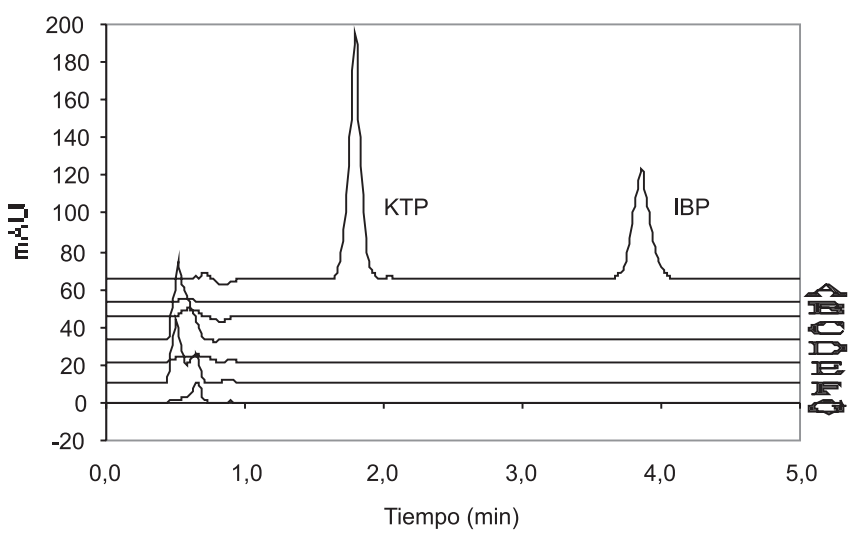

Figura 4. Cromatogramas que indican la especificidad del método analítico. A: IBP (300 $\left.\mathrm{g} \mathrm{mL}^{-1}\right)$ y KTP (estándar interno) en el diluente; B: Fase móvil; $C$ : Diluente; D: Solución amortiguadora pH 5,0; E: Solución amortiguadora pH 6,5; F: Medio FeSSIF; G. Medio FaSSIF

aparece la señal que corresponde al IBP o al KTP. En general, se observaron disminuciones notorias en el área del pico de IBP en las muestras preparadas en el medio FaSSIF cuando se adicionó $\mathrm{HCl}$ y $\mathrm{NaOH} 1,0 \mathrm{M}$ que correspondieron al 32 y $28 \%$, respectivamente, respecto al estándar sin degradar.

Cuando la muestra en el medio FaSSIF se expuso a peróxido de hidrógeno al $30 \%$, al medio acuoso a $60,0^{\circ} \mathrm{C}$, y a la radiación UV sufrió poca degradación ( 8,5 y 3,5\%, respectivamente). En cuanto al medio FeSSIF, se aprecia que el IBP fue degradado en mayor proporción cuando se expuso a hidrólisis en medio ácido y bajo radiación UV (28 y 18\%, respectivamente), y en menor proporción en condiciones oxidativas (4\%). La Tabla 3 indica las áreas obtenidas en cada caso.

Tabla 3. Áreas de estándar de IBP y de las muestras expuestas a condiciones de degradación

\begin{tabular}{lcc}
\hline Condiciones de degradación & \multicolumn{2}{c}{ Áreas / Medios biorrelevantes } \\
& FaSSIF & FeSSIF \\
\hline $1 \mathrm{~mL} \mathrm{HCl} \mathrm{1,0} \mathrm{M}$ & 401,7 & 423,8 \\
$1 \mathrm{~mL} \mathrm{NaOH} \mathrm{1,0} \mathrm{M}$ & 419,9 & 585,6 \\
$1 \mathrm{~mL} \mathrm{H}_{2} \mathrm{O}_{2} 30 \%$ & 542,2 & 564 \\
$1 \mathrm{~mL} \mathrm{H}_{2} \mathrm{O}$ & 562,5 & 578,7 \\
luz UV $\lambda 254 \mathrm{~nm}$ & 566,9 & 482,5 \\
$\mathrm{Blanco} \mathrm{H}^{+} / \mathrm{OH}^{-}$ & 0 & 0 \\
Blanco $\mathrm{H}_{2} \mathrm{O}_{2} 30 \%$ & 0 & 0 \\
Área estándar & 588 & \\
\hline
\end{tabular}

Las curvas de calibración fueron realizadas para el sistema, las soluciones amortiguadoras y los medios biorrelevantes, con el fin de establecer la regresión entre la relación de áreas del IBP y del estándar interno $(y)$ y la concentración del IBP $(x)$. La Figura 5 muestra las curvas de calibración obtenidas en los sistemas empleados.

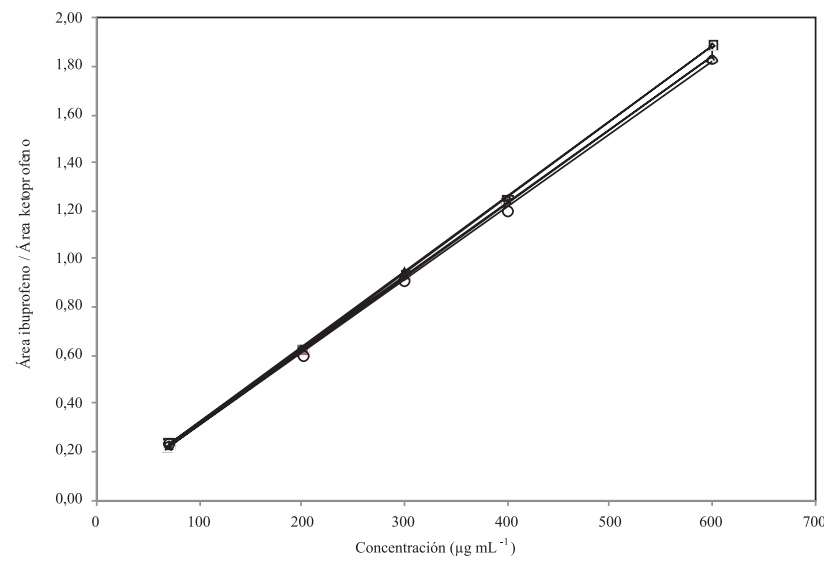

Figura 5. Curvas de calibración de IBP en diluente y los medios empleados. (०): Sistema; $(\Delta)$ : Solución amortiguadora pH 5,0; (+): Solución amortiguadora $\mathrm{pH}$ 6,5; $(\diamond)$ : FeSSIF; (口): FaSSIF

Los resultados del análisis de la linealidad se muestran en la Tabla 4. Se puede apreciar que los valores experimentales de $t$ para el intercepto son menores que los valores tabulados, demostrando que los interceptos en todas las curvas de calibración no son significativamente diferentes de cero. Con respecto a las pendientes se encuentran valores experimentales de $t$ mayores a los tabulados, indicando con esto que las pendientes son significativamente diferentes a cero. La significancia de la regresión se confirma a través de los valores experimentales de $F$, puesto que son en magnitud mayor al respectivo valor de $F$ tabulado. Los coeficientes de correlación de las curvas de calibración oscilan entre 0,99947 y 0,99985, indicando que los datos experimentales se ajustan al modelo de regresión lineal.

La Tabla 5 muestra los resultados obtenidos para evaluar la precisión de cada uno de los métodos. Los resultados de la repetibilidad tanto para el sistema como para el método, indican una desviación estándar relativa con valores que oscilan entre 0,45 y $1,95 \%$ cuando se emplean 3 réplicas a 3 niveles de concentración. La farmacopea USP $30^{13}$ y el manual de la $\mathrm{AOAC}^{17}$ en general permiten coeficientes de variación menores a $2 \%$, de modo que la repetibilidad del método cumple con estas especificaciones. En el caso de la precisión intermedia, los resultados de los análisis de varianzas indican con una confianza del $95 \%$ que no existe diferencia significativa en los resultados obtenidos entre los analistas en días diferentes y réplicas distintas, empleando el diluente, las soluciones amortiguadoras y los medios biorrelevantes. Además las réplicas muestran coeficientes de variación entre 0,44 y 2,1\%. El método cumple con el parámetro de precisión.

La exactitud del método fue determinada al utilizar un estándar de IBP de concentración conocida. La Tabla 6 muestra los resultados obtenidos. En general se aprecia que la exactitud se encuentra entre 98,10 y $106,67 \%$ dependiendo del medio empleado. Los coeficientes de variación se encuentran entre 0,55 y $2,30 \%$.

Tabla 4. Resultados del análisis de varianza y los estadísticos de prueba para la evaluación del parámetro de linealidad

\begin{tabular}{|c|c|c|c|c|c|c|c|c|}
\hline \multicolumn{9}{|c|}{ Parámetros } \\
\hline & Ecuación & $r$ & $n$ & $F_{\text {exp }}$ & $F_{\text {tab }}$ & $\begin{array}{l}t_{\text {exp }} \\
\text { pendiente }\end{array}$ & $\begin{array}{l}t_{\text {exp }} \\
\text { intercepto }\end{array}$ & $t_{\text {tab }}$ \\
\hline Sistema & $y=3,012(0,018) \cdot 10^{-3} x+0,012(0,006)$ & 0,99968 & 20 & 28743,47 & 4,54 & 169,53 & 1,81 & 2,10 \\
\hline $\mathrm{B} \mathrm{pH} 5,0$ & $y=3,054(0,028) \cdot 10^{-3} x+0,013(0,010)$ & 0,99947 & 15 & 12269,93 & 4,96 & 110,77 & 1,34 & 2,16 \\
\hline $\mathrm{B}$ pH 6,5 & $y=3,064(0,012) \cdot 10^{-3} x+0,007(0,004)$ & 0,99985 & 20 & 61306,22 & 4,54 & 247,60 & 1,64 & 2,10 \\
\hline FeSSIF & $y=3,125(0,021) \cdot 10^{-3} x+0,005(0,007)$ & 0,99961 & 20 & 22854,16 & 4,54 & 151,18 & 0,73 & 2,10 \\
\hline FaSSIF & $y=3,118(0,024) \cdot 10^{-3} x+0,011(0,008)$ & 0,99951 & 19 & 17278,02 & 4,60 & 131,45 & 1,39 & 2,11 \\
\hline
\end{tabular}

$\mathrm{B}=$ Solución amortiguadora, $r=$ coeficiente de correlación, $n=$ Número de datos, $t_{\mathrm{exp}}=$ Estadístico experimental para pendiente o intercepto, $t_{\mathrm{tab}}=$ Estadístico tabulado 
Tabla 5. Repetibilidad y precisión intermedia del método analítico

\begin{tabular}{|c|c|c|c|c|c|c|c|}
\hline & \multicolumn{6}{|c|}{ Repetibilidad } \\
\hline & & & Sistema & B pH 5,0 & B pH 6,5 & FeSSIF & FaSSIF \\
\hline \multirow{3}{*}{\multicolumn{2}{|c|}{$\mathrm{X}$}} & B & 68,66 & 73,08 & 74,81 & 71,16 & 72,46 \\
\hline & & M & 301,60 & 301,38 & 298,42 & 297,11 & 298,87 \\
\hline & & A & 603,57 & 605,66 & 606,81 & 596,12 & 606,02 \\
\hline \multirow{3}{*}{\multicolumn{2}{|c|}{ SD }} & B & 0,31 & 1,42 & 0,82 & 0,43 & 1,03 \\
\hline & & M & 4,92 & 3,45 & 5,35 & 1,42 & 3,35 \\
\hline & & A & 6,56 & 10,57 & 8,57 & 6,39 & 6,24 \\
\hline & RSD (\%) & B & 0,45 & 1,95 & 1,09 & 0,60 & 1,42 \\
\hline & & M & 1,63 & 1,15 & 1,79 & 0,48 & 1,12 \\
\hline & & A & 1,09 & 1,75 & 1,41 & 1,07 & 1,03 \\
\hline & & \multicolumn{6}{|c|}{ Precisión intermedia } \\
\hline \multirow[t]{6}{*}{ Día 1} & 1 & $\mathrm{X}$ & 299,56 & 299,33 & 298,42 & 302,09 & 298,45 \\
\hline & & SD & 1,56 & 2,80 & 5,35 & 2,96 & 3,27 \\
\hline & & $\operatorname{RSD}(\%)$ & 0,52 & 0,94 & 1,79 & 0,98 & 1,10 \\
\hline & 2 & $\mathrm{X}$ & 300,65 & 304,51 & 306,14 & 300,35 & 299,53 \\
\hline & & $\mathrm{SD}$ & 5,51 & 2,65 & 2,26 & 2,61 & 0,96 \\
\hline & & $\operatorname{RSD}(\%)$ & 1,83 & 0,87 & 0,74 & 0,87 & 0,32 \\
\hline \multirow[t]{9}{*}{ Día 2} & 1 & $\mathrm{X}$ & 301,60 & 303,83 & 303,64 & 293,64 & 298,87 \\
\hline & & SD & 4,92 & 3,75 & 5,97 & 6,18 & 3,35 \\
\hline & & $\operatorname{RSD}(\%)$ & 1,63 & 1,23 & 1,97 & 2,10 & 1,12 \\
\hline & 2 & $X$ & 296,35 & 302,88 & 303,19 & 299,92 & 299,77 \\
\hline & & SD & 1,67 & 3,30 & 6,12 & 5,25 & 1,31 \\
\hline & & RSD (\%) & 0,56 & 1,09 & 2,02 & 1,75 & 0,44 \\
\hline & $F$ Día & & 0,23 & 0,59 & 0,11 & 2,97 & 0,09 \\
\hline & $F$ Analista & & 0,80 & 1,29 & 1,12 & 0,77 & 0,78 \\
\hline & $F$ Réplica & & 1,08 & 1,67 & 0,22 & 1,80 & 3,07 \\
\hline
\end{tabular}

$\mathrm{B}=$ Solución amortiguadora, $\mathrm{SD}=$ Desviación estándar, $\mathrm{RSD}=$ Desviación estándar relativa, $\mathrm{X}=$ Promedio de la concentración $\left(\mu \mathrm{g} \mathrm{mL}^{-1}\right), \mathrm{B}=70 \mu \mathrm{g}$ $\mathrm{mL}^{-1}, \mathrm{M}=300 \mu \mathrm{g} \mathrm{mL}^{-1}, \mathrm{~A}=600 \mu \mathrm{g} \mathrm{mL}^{-1}, 1=$ Analista $1,2=$ Analista $2, F_{\text {tabla-dia }}$ $(1 / 11,0,05)=4,84, F_{\text {tabla-analista }}(1 / 11,0,05)=4,84, F_{\text {tabla-réplica }}(2 / 11,0,05)=3,98$

Tabla 6. Resultados obtenidos para la evaluación del parámetro de exactitud del método

\begin{tabular}{lccccc}
\hline & & $\mathrm{B} \mathrm{pH} \mathrm{5,0}$ & $\mathrm{B} \mathrm{pH} \mathrm{6,5}$ & FeSSIF & FaSSIF \\
\hline Recuperación & $\mathrm{B}$ & 98,10 & 99,91 & 106,67 & 106,43 \\
$(\%)$ & $\mathrm{M}$ & 102,70 & 102,00 & 101,51 & 101,40 \\
& $\mathrm{~A}$ & 102,39 & 101,11 & 100,65 & 104,53 \\
RSD (\%) & $\mathrm{B}$ & 1,40 & 2,30 & 1,19 & 0,86 \\
& $\mathrm{M}$ & 1,37 & 0,55 & 2,09 & 1,97 \\
& $\mathrm{~A}$ & 1,72 & 1,20 & 1,38 & 1,02 \\
\hline
\end{tabular}

$\mathrm{B}=$ Solución amortiguadora, $\mathrm{RSD}=$ Desviación estándar relativa, $\mathrm{B}=70$ $\mu \mathrm{g} \mathrm{mL} \mathrm{mL}^{-1}, \mathrm{M}=300 \mu \mathrm{g} \mathrm{mL}-1, \mathrm{~A}=600 \mu \mathrm{g} \mathrm{mL} \mathrm{m}^{-1}$.

Los límites de cuantificación y detección fueron determinados a través de la pendiente y la desviación estándar del intercepto de cada una de las curvas de calibración. En la Tabla 7 se aprecian estos resultados.

Se demostró estabilidad aceptable del analito bajo las condiciones ensayadas. La recuperación del estándar de IBP a temperatura ambiente por $96 \mathrm{~h}$, a $4,0{ }^{\circ} \mathrm{C}$ por $96 \mathrm{~h}$ y a $40,0{ }^{\circ} \mathrm{C}$ por $48 \mathrm{~h}$ en el diluente fue de $98,89,99,60$ y 97,83\%, respectivamente. Las muestras de IBP
Tabla 7. Límites de detección (LD) y cuantificación (LC) del método analítico

\begin{tabular}{lcc}
\hline & $\mathrm{LD}(\mu \mathrm{g} \mathrm{mL}-1)$ & $\mathrm{LC}\left(\mu \mathrm{g} \mathrm{mL}{ }^{-1}\right)$ \\
\hline Sistema & 7,04 & 21,35 \\
B pH 5,0 & 10,78 & 32,67 \\
B pH 6,5 & 4,82 & 14,62 \\
FeSSIF & 7,90 & 23,94 \\
FaSSIF & 8,66 & 26,24 \\
\hline
\end{tabular}

en la solución amortiguadora de $\mathrm{pH}$ 5,0 presentaron recuperaciones de 100,90, 108,04 y 102,93\% y en la solución amortiguadora de $\mathrm{pH}$ 6,5 de 98,$02 ; 98,53$ y 98,81\%. En el medio FeSSIF la recuperación de IBP fue de 106,$40 ; 105,03$ y 102,73\%, mientras que en el medio FaSSIF esta recuperación fue de 97,13; 96,09 y 97,21\% bajo las mismas condiciones. En todas las muestras el cambio del porcentaje de activo fue menor al 10\%. Las desviaciones estándar relativas en todos los ensayos de estabilidad variaron entre 0,30 y $2,31 \%$.

Una vez que fue validado el método analítico, se determinó la solubilidad de IBP en los medios FaSSIF y FeSSIF y las soluciones amortiguadoras de $\mathrm{pH} 6,5$ y 5,0 a la temperatura de $37^{\circ} \mathrm{C}$. Los resultados son similares a los encontrados por otros autores (Tabla 8). Levis y colaboradores reportaron solubilidades de 1,56 y $1,16 \mathrm{mg}$ $\mathrm{mL}^{-1}$ para el medio FaSSIF y FeSSIF, respectivamente. ${ }^{18}$ Además, se aprecia un incremento de 4,1 veces en la solubilidad de IBP en el medio FeSSIF con respecto a la solución amortiguadora de $\mathrm{pH}$ 5,0; este incremento se debe a la solubilización del IBP en las micelas mixtas de taurocolato de sodio y fosfatidilcolina, tal y como se aprecia en otros antiinflamatorios no esteroidales. ${ }^{4,19}$ Sin embargo, no se observaron cambios en el orden de magnitud de la solubilidad de IBP obtenida en FaSSIF con respecto a la solubilidad en la solución amortiguadora de $\mathrm{pH}$ 6,5. Esto último indica que el $\mathrm{pH}$ tiene un efecto mayor que la presencia de los surfactantes en la solubilidad, bajo estas condiciones. ${ }^{19}$

Tabla 8. Solubilidad de IBP en los medios biorrelevantes y en las soluciones amortiguadoras a $37,0 \pm 0,1^{\circ} \mathrm{C}$ expresada en $\mathrm{mg} \mathrm{mL}^{-1}$ ( \pm desviación estándar)

\begin{tabular}{lccc}
\hline $\operatorname{IBP}\left(\mathrm{mg} \mathrm{mL}^{-1}\right)$ & & & \\
\hline & $\begin{array}{c}\text { Solución } \\
\text { amortiguadora }\end{array}$ & & $\begin{array}{c}\text { Solución } \\
\text { amortiguadora }\end{array}$ \\
FaSSIF & $\mathrm{pH} 6,5$ & FeSSIF & $\mathrm{pH} \mathrm{5,0}$ \\
$1,592(0,020)$ & $1,490(0,007)$ & $1,285(0,015)$ & $0,306(0,006)$ \\
\hline
\end{tabular}

Según la Organización Mundial de la Salud (OMS) en el Informe $40,{ }^{9} \mathrm{y}$ basados en el SCB, se ha establecido que un fármaco presenta alta solubilidad cuando la relación Dosis/Solubilidad es menor a $250 \mathrm{~mL}$ cuando esta es medida las soluciones amortiguadoras de $\mathrm{pH}$ 1,$2 ; 4,5$ y 6,8 . Con los resultados obtenidos se determinó la relación entre la dosis máxima del IBP establecida en la lista de medicamentos esenciales de la OMS (400 mg) y la solubilidad experimental, encontrando volúmenes de 251,6; 268,4; 312,5 y $1290 \mathrm{~mL}$, en los medios FaSSIF, la solución amortiguadora de pH 6,5, FeSSIF y la solución amortiguadora de $\mathrm{pH}$ 5,0, respectivamente. Según esto, si la solubilidad se hiciera en los medios biorrelevantes este fármaco podría pertenecer a la Clase I y no a la II.

Se han sugerido varias modificaciones al SCB, entre las cuales se encuentran el uso de medios biorrelevantes que simulen las condiciones fisiológicas del tracto intestinal para la determinación de la solubilidad y la disolución, ${ }^{8}$ incrementar el volumen a un valor de 500 $\mathrm{mL}$ para definir la relación dosis/solubilidad, ${ }^{7}$ entre otras. Asumiendo un incremento a $500 \mathrm{~mL}$ en el volumen límite de clasificación de solubilidad, el IBP estaría clasificado en la Clase I si el ensayo se realizara con los medios FaSSIF y FeSSIF. 
En el caso del IBP, a pesar de que se encuentre en la Clase II, se ha incluido dentro de los compuestos cuyos productos son susceptibles de bioexención de los estudios de bioequivalencia. ${ }^{11}$ Muchos compuestos que están actualmente en la Clase II pueden presentar incrementos en la solubilidad si se usan los medios biorrelevantes, lo que haría pensar que pueden hacer parte de la Clase I según el SCB y por consiguiente los compuestos de liberación oral inmediata que los contienen serían candidatos a la bioexención, teniendo en cuenta los lineamientos de las entidades regulatorias.

\section{CONCLUSIONES}

El método analítico por CLAE es adecuado para la cuantificación de IBP en medios biorrelevantes y en las soluciones amortiguadoras de $\mathrm{pH}$ 6,5 y 5,0, en términos de los diferentes parámetros analíticos evaluados. El tiempo de análisis es relativamente corto, lo que facilita su uso en los estudios termodinámicos de solubilidad y solubilización de IBP. La determinación de la solubilidad aparente en medios como FeSSIF y FaSSIF, de sustancias que presentan valores de solubilidad bajos en el agua permite obtener valores semejantes a los que se presentarían bajo condiciones in vivo en el intestino. La utilización de los medios biorrelevantes es una herramienta que puede aplicarse en la clasificación de compuestos como IBP y otros compuestos lipófilos neutros de la Clase II según el SCB. Finalmente se puede mencionar que el método analítico propuesto podría ser utilizado para desarrollar estudios termodinámicos de solubilización de este fármaco en estos medios simulados, lo cual sería de amplio interés farmacéutico y químico, puesto que podrían contribuir al entendimiento del efecto de las diferentes características fisicoquímicas del ambiente biológico del tracto gastrointestinal sobre la absorción de fármacos.

\section{AGRADECIMIENTOS}

A la Dirección de Investigación (DIB) de la Universidad Nacional de Colombia (UNC), por la financiación del presente proyecto, y al Departamento de Farmacia de la UNC por facilitar los equipos e instalaciones para el desarrollo de este trabajo.

\section{REFERENCIAS}

1. Löbenberg, R.; Vieira, M.; Amidon, G. L. En Oral Drug Absorption. Prediction and Assessment; Dressman, J. B.; Lennernäs, H., eds.; Marcel Dekker, Inc.: New York Basel, 2000, p. 137.

2. Müllertz, A. En Solvent Systems and Their Selection in Pharmaceutics and Biopharmaceutics; Augustijns, P.; Brewster, M. E., eds.; Springer and AAPS Press: New York, 2007, vol. 6, p. 151.
3. Dressman, J. B.; Vertzoni, M.; Goumas, K.; Reppas, C.; Adv. Drug Deliv. Rev. 2007, 59, 591.

4. Glomme, A.; Marz, J.; Dressman, J. B. En Pharmacokinetic Profiling in Drug Research. Biological, Physicochemical, and Computational Strategies; Testa, B.; Krämer, S. D.; Wunderli-Allenspach H.; Folkers, G., eds.; Wiley-VCH, Zürich, 2006, p. 259.

5. Dressman, J. B.; Amidon, G. L.; Reppas, C.; Shah, V. P.; Pharm. Res. 1998, $15,11$.

6. Galia, E.; Nicolaides, E.; Horter, D.; Lobenberg, R.; Reppas, C.; Dressman, J. B.; Pharm. Res. 1998, 15, 698.

7. Yu, X. L.; Amidon, G. L.; Polli, J. E.; Zhao, H.; Mehta, M. U.; Conner, D. P.; Shah, V. P.; Lesko, L. J.; Chen, M-L.; Lee, V. H. L.; Hussain, A. S.; Pharm. Res. 2002, 19, 921; Fagerholm, U.; J. Pharm. Pharmacol. 2007, 59, 751 .

8. Yazdanian, M.; Briggs, K.; Jankovsky, C.; Hawi, A.; Pharm. Res. 2004, 21, 293.

9. WHO Expert Committee on Specifications for Pharmaceutical Preparations; WHO Technical Report Series, Fortieth Report No 937, Annex 7, Geneva, 2006, p. 358-413.

10. Budavari, S.; O'Neil, M. J.; Smith, A.; Heckelman, P. E.; Obenchain Jr., J. R.; Gallipeau, J. A. R.; D'Arecea, M. A.; The Merck Index: An Encyclopedia of Chemicals, Drugs, and Biologicals, $13^{\text {th }}$ ed., Merck \& Co., Inc.: Whitehouse Station, 2001.

11. Potthast, H.; Dressman, J. B.; Juginger, H. E.; Midha, K. K.; Oeser, H.; Shah, V. P.; Vogelpoel, H.; Barends, D. M.; J. Pharm. Sci. 2005, 94 , 2121.

12. Sultan, M.; Stecher, G.; Stoggl, W. M.; Bakry, R.; Zaborski, P.; Huck, C. W.; El Kousy, N. M.; Bonn, G. K.; Curr. Med. Chem. 2005, 12, 573; Sena, M. M.; Freitas, C. B.; Silva, L. C.; Perez, C. N.; de Paula, Y. O.; Quim. Nova 2007, 30, 75; Thomas, A. B.; Dumbre, N. G.; Nanda, R. K.; Kothapalli, L. P.; Chaudhari, A. A.; Deshpande, A. D.; Chromatographia 2008, 68, 843 .

13. US Pharmacopeia, $30^{\text {th }}$ revision; United States Pharmacopeial Convention, Inc.: Rockville, 2007, p. 680.

14. Farrar, H.; Letzig, L.; Gill, M.; J. Chromatogr., B: Anal. Technol. Biomed. Life Sci. 2002, 780, 341.

15. Vertzoni, M.; Fotaki, N.; Kostewicz, E.; Stippler, E.; Leuner, C.; Nicolaides, E.; Dressman, J.; Reppas, C.; J. Pharm. Pharmacol. 2004, 56, 453.

16. ICH, Food and Drug Administration, U.S. Department of Health and Human Services; Guidance for Industry: Q2B Validation of Analytical Procedures: Methodology, Rockville, 1996.

17. Association of Official Analytical Chemists; Statistical Manual of the $A O A C, 12^{\text {th }}$ ed.; AOAC: Washington, 1975, p. 1094.

18. Levis, K. A.; Lane, M. E.; Corrrigan, O. I.; Int. J. Pharm. 2003, 253, 49.

19. Tenhoor, C. N.; Bakatselou, V.; Dressman, J. B.; Pharm. Res. 1991, 8, 1203. 